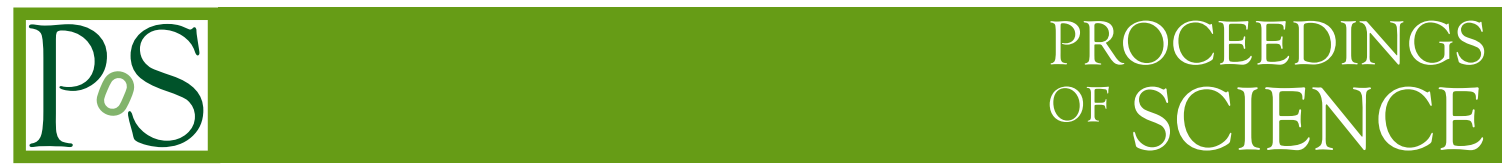

\title{
Multifrequency astrophysics with very small satellites
}

\author{
René Hudec ${ }^{1,2 *}$ \\ ${ }^{1}$ Czech Technical University in Prague, Faculty of Electrical Engineering, \\ Technická 2, 16000 Prague, Czech Republic \\ ${ }^{2}$ Engelhardt Astronomical observatory, Kazan Federal University, Kremlyovskaya street 18, \\ 420008 Kazan, Russia \\ hudecren@fel.cvut.cz
}

The recent progress in picosatellites technology, allows to consider their use for astrophysics, based on miniaturized scientific payload. We suggest possible astrophysical payloads for cubesats with emphasis on high-energy and UV astrophysics.

XII Multifrequency Behaviour of High Energy Cosmic Sources Workshop 12-17 June, 2017

Palermo, Italy

\footnotetext{
* Speaker.
} 


\section{Introduction}

The recent situation in experimental satellite astronomy and astrophysics is affected by funding problems. For example, there is very large competition for ESA M and L missions, with typically several dozens proposals for one call delivered.

On the other hand, in the recent years, there was essential improvement in cubesats technology, allowing scientific payloads for cubesats to be considered. The miniature satellites are recently in development at many institutes and universities, mostly with a participation of students. The fast development of the related techniques and technologies enables to consider small scientific payloads for these satellites.

The pico and nanosatellites are recently in development at many universities, mostly with involvement of students and education. The CubeSat standard size is 1 Liter Volume, i.e. $10 \times 10 \times$ $10 \mathrm{~cm}$, and a typical weight is $1.3 \mathrm{~kg}$, but multiple modules are possible, i.e. $3 \mathrm{U}=3$ modules/units, i.e. $10 \times 10 \times 30 \mathrm{~cm}$, typically up to $12 \mathrm{U}$.

\section{Scientific payload for pico and nanosatellite}

In this paper, we briefly discuss few ideas for application of pico and cubesatellites in highenergy and space astronomy and astrophysics. We note that these payloads must meet the following strict requirements for the scientific payload for picosatellites. They must fit into a small volume, typically $30 \times 10 \times 10 \mathrm{~cm}$ or less ( $3 \mathrm{U}$, i.e. 3 cubesat modules), they must be of low weight, typically less than $1 \mathrm{~kg}$, with low power consumption of about 10 Watts or less.

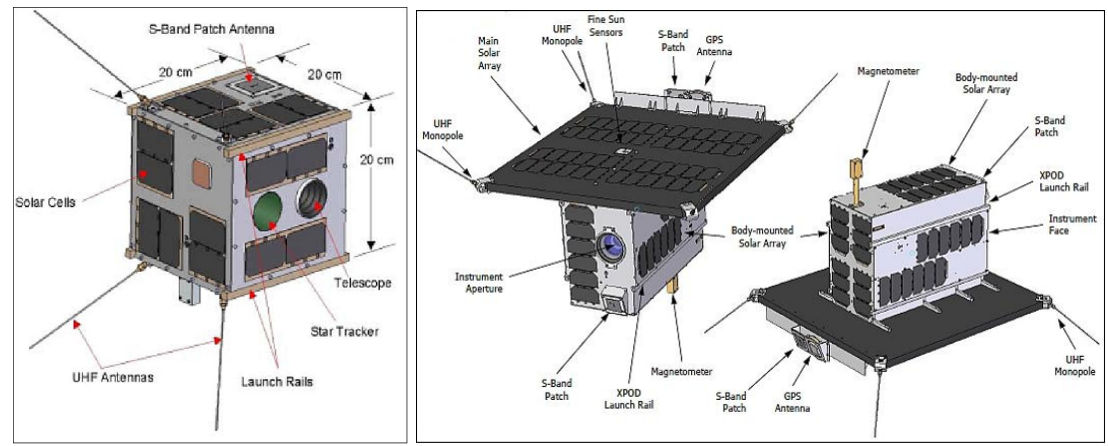

Figure 1: BRITE X 8U CubeSat (left) and Small scientific 16U satellite BRITE CZ with combined payload UV-BRITE and X-BRITE (right)

\section{Payload example 1: Miniature X-ray monitor}

The Lobster Eye (LE) X-ray optics was originally proposed by Schmidt (1975) and Angel (1979). Since then, numerous test specimens of Lobster Eye telescopes were designed and tested (e.g. Inneman et al. 1999; Hudec et al. 2000, 2003, 2004; Tichý et al. 2009, 2011). The LobsterEye (LE) X-ray telescope can be miniaturized for an application in picosatellites. The LE telescopes are novel wide field X-ray telescopes with the field of view (FOV) of 100 sq. deg. They are 
more easily possible (a classical X-ray optics has the FOV of only 1 deg or less) and are based on a real analogy with the lobster eyes.

The LE payload for picosatellites also requires miniaturized focal detectors. The best available option for the author team of this paper is the detector Medipix (Timepix). Medipix is a family of photon counting pixel detectors developed by an international collaboration, hosted by CERN. The CTU in Prague is a member of this cooperation. The Medipix detector represents a suitable imaging detector for a use in space LE telescopes.

However, the following items are important: 1 . The detector is not yet space qualified; 2 . Its spectral coverage starts only in energies above $3 \mathrm{keV}$, while the spectral coverage of the LE optics in the Schmidt arrangement is typically from the visible light up to the energies of 8 or $10 \mathrm{keV}$.

However, a more suitable spacecraft is a $6 \mathrm{U}$ CubeSat as it allows to accommodate several LE modules to increase the final FOV (Tichý et al. 2013a). We elaborated a design of a new SLE prototype as follows. More mirrors: 333 per set, a larger input area $(10 \times 10 \mathrm{~cm})$, a special coating to increase the reflectivity at higher energies, a better manufacturing technology, the focal length kept at $250 \mathrm{~mm}$.
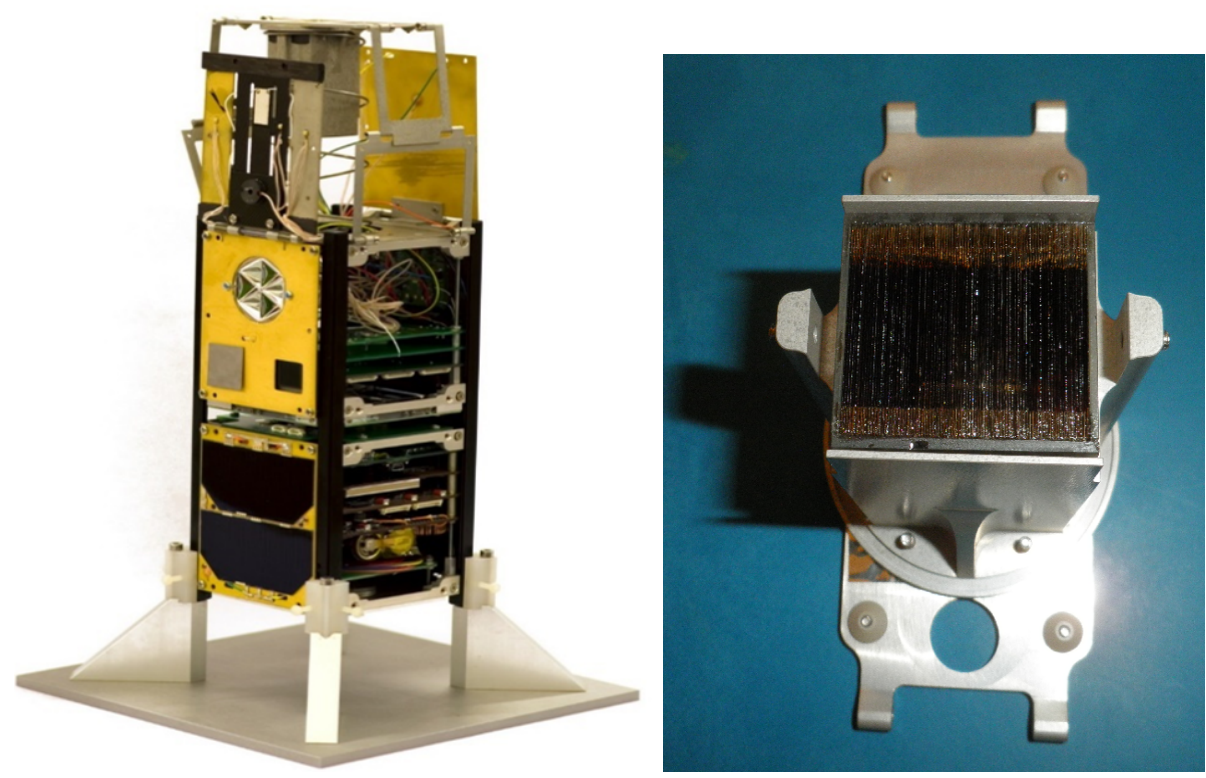

Figure 2: The VZLUSAT1 2U cubesatellite (left) and the 1D LE optics module onboard (right), Urban et al. 2017a, 2017b, Daniel et al., 2016.

The 1st LE X-ray monitor was launched into Earth orbit in June 23, 2017, onboard small Czech 2U cubesatellite VZLUSAT1 (Figs. 1 and 2, Urban et al. 2017a, 2017b, Daniel 2016). The small X-ray experiment is based on 1D LE X-ray optics module and on Timepix detector used to record the focal images. The main goal is the technology demonstration in orbit, with potential of future larger experiments based on analogous hardware. The 1D LE is more suitable for low X-ray intensities and also harder X-rays $(5-20 \mathrm{keV})$. Optics above $35 \mathrm{keV}$ behaves as a collimator (Soller slit), so that the gain is only 1 . Maximum energy is limited by the Timepix detector (3-50 keV). Timepix can be used also for measurement of spectrum of astrophysical objects. The designed 
FOV is $3 \times 3$ deg.

\subsection{Science objectives}

Wide field X-ray monitors of Lobster Eye type were demonstrated to play an important role in modern astrophysics (e.g. Hudec et al. 2007; Švéda et al. 2004). The most important scientific cases are briefly summarized below. (i) A long-term (months) measurement of the light curves of bright persistent X-ray binaries in the direction toward the center of the Galaxy in the soft X-ray band. (ii) Detection and measurement of the light curves of bright transient events of X-ray binaries in the direction toward the center of the Galaxy in the soft X-ray band.

\subsection{Modes of Operation}

The LE telescopes can typically serve in two basic operation modes as follows. (i) Starrying (pointed) mode - only for satellite with pointing, and (ii) Scanning mode (no satellite pointing and/or stabilization required). We note that many cubesats do not have any stabilization suitable for pointed observations.

\section{Example 2: Miniature UV experiment}

Also UV telescopes can be miniaturized for cubesat applications. For example, for the proposed BRITE UV cubesat, the payload is based on the miniature UV spectrographic camera, significantly upgraded camera system which was used by NASA in Gemini (Henize et al., 1968) and Skylab UV experiments led by Prof. Karl G. Henize (Figs. 3 and 4).

For Gemini UV experiments, the used UV lens had aperture $22 \mathrm{~mm}, \mathrm{f}=73 \mathrm{~mm}, \mathrm{f} / 3.3$, and FOV 30 degrees, with working range $2300-5000 \mathrm{~A}$. Grating was used with $184 \mathrm{~A} / \mathrm{mm}$ at $2000 \mathrm{~A}, 600$ lines/mm as well as prism $1400 \mathrm{~A} / \mathrm{mm}$ at $2500 \mathrm{~A}$. Gemini XI, XII experiments used a prism, while Gemini X, XI and XII experiments used a grating, all these experiments were operated during EVA (outside spacecraft).

The Skylab UV experiment was larger, based f/3 Ritchey-Chretien telescope with aperture 15 $\mathrm{cm}$ and FOV of 4x5 deg, equipped with prism with dispersion of 64, 365 and $1281 \mathrm{~A}$ at 1400, 2000, and $2800 \mathrm{~A}$, and spectral resolution of 2, 12, and $41 \mathrm{~A}$.

The scientific objective of the proposed cubesat UV experiment is analogous to he historical UV experiments on Gemini (Morgan et al., 1975) and Skylab (Ocalaghan et al. 1978), namely to obtain line spectra in the wavelength 2200 to $4000 \mathrm{~A}$ of stars. The system will be represented by a UV camera with a UV lens with a $30 \mathrm{~mm}$ aperture, $100 \mathrm{~mm}$ focal length and a field of 30 degrees in diameter (preliminary values). CMOS or cooled CCD detector will be used as an image detector in the focal plane. As an alternative, an objective grating will be used to produce a dispersion of 180 Angstroms per mm at $2000 \mathrm{~A}$. In addition, the quartz objective prism which produces a dispersion of 1400 Angstroms per mm at $2500 \mathrm{~A}$ is considered to be used.

The possible platform for the UV experiment is $3 \mathrm{U}$ to $8 \mathrm{U}$ cubesat with deploy-able mechanisms to extend length and UV lens or folded reflector with aperture 3 to $10 \mathrm{~cm}$. sCMOS detector, eg e2V1 CIS101-00-M05 chip, can serve as possible focal detector. This device has 1415 (H) x 1430 (V) pixels each $14.81 \mu \mathrm{m} \times 11.53 \mu \mathrm{m}$ in size. The objective prism and/or grating should be included for spectroscopy. 


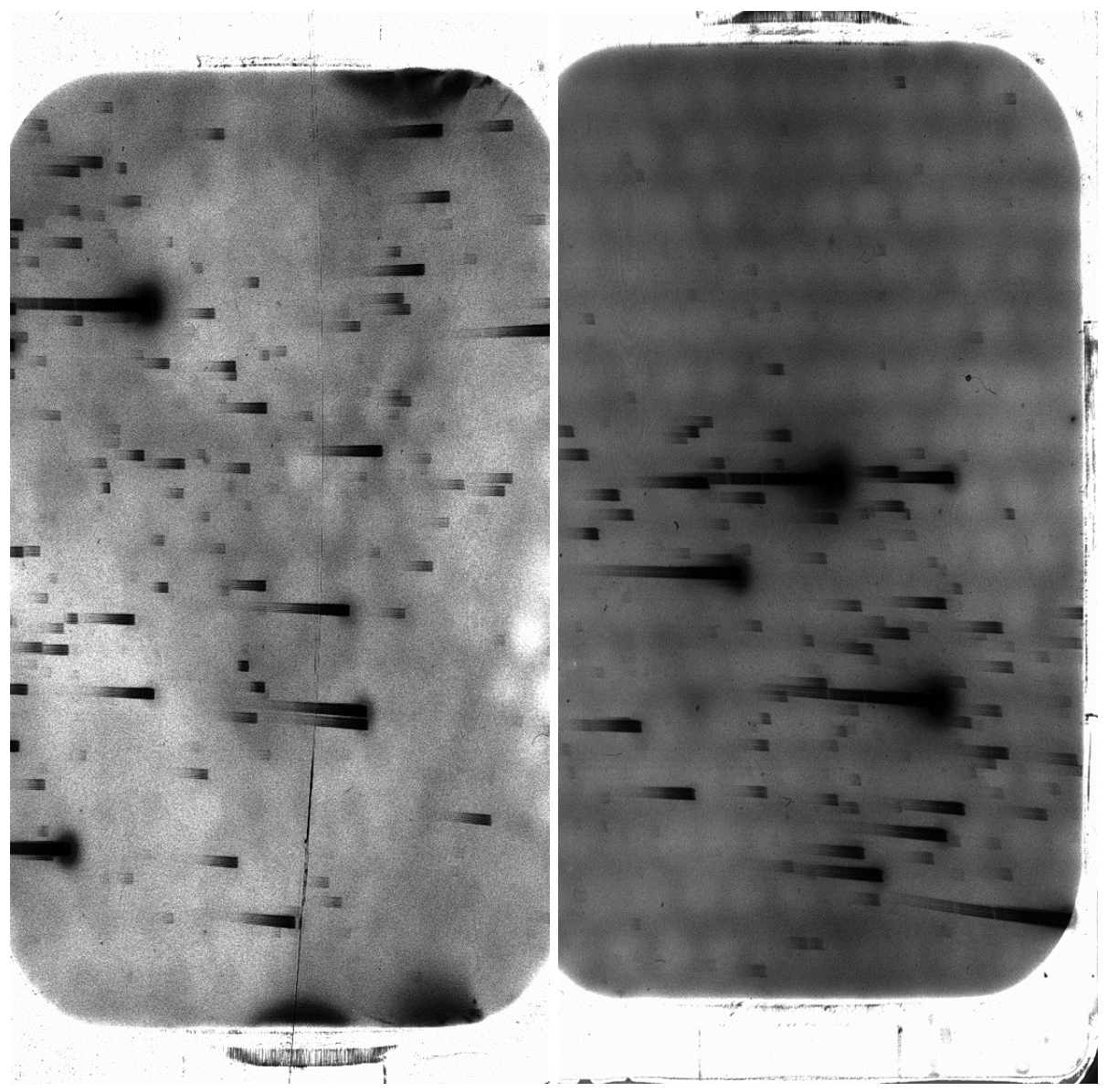

Figure 3: New scans performed in 2016 from original Skylab UV expriment films.

If compared with the Skylab UV experiment, the main difference is that the recording medium will be CMOS instead of film, hence with essential increase in sensitivity (e.g. for Skylab, the magnitude limit was 9 mag lim mag, compared to BRITE UV estimated limit of 12 mag).

\section{Conclusions}

The proposed mission (miniature LE X-ray telescope/monitor in a picosatellite) can acquire scientifically important data for a low price. The optics for the presented mission is feasible. The scientific justification is strong, including several perspective areas of modern astrophysics. A new prototype of the optics (based on the optimized design for a picosatellite) was already developed and tested. Also for UV astrophysics, miniature UV telescope/spectrograph for cubesat application could be considered as well.

We also note additional, more advanced science potential of using network of identical CubeSats for particular scientific tasks. This is potential area where CubeSats can compete with larger missions.

Acknowledgments This work was supported by the grant GA CR 13-33324S. 


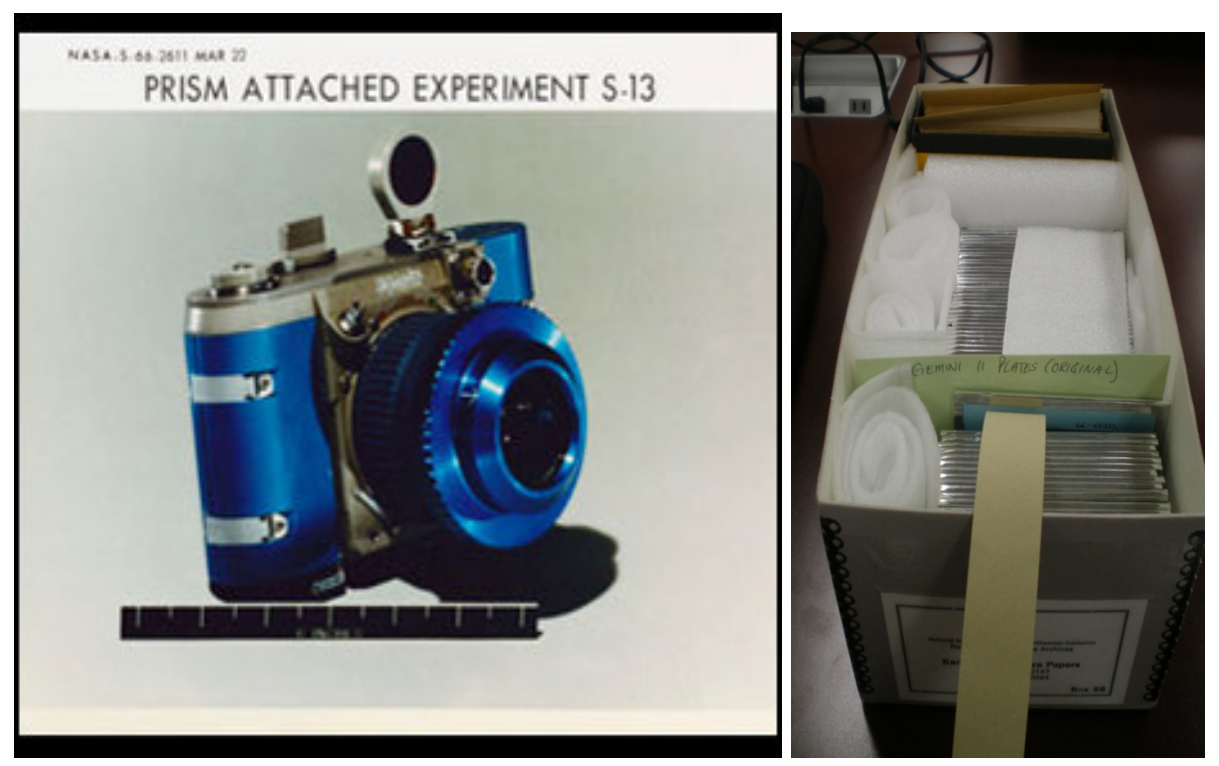

Figure 4: Prof. K. Henize Gemini UV experiment (left) and the photographic plate archive with these data (right).

\section{References}

[1] Henize, K. G., J. D. Wray, and L. R. Wackerling: Ultraviolet objective-prism spectra from Gemini XI and XII manned space flights., Bulletin of the Astronomical Institutes of Czechoslovakia, Volume 19, id.279 (1968)

[2] Hudec, R., et al., Proc. SPIE 5488, UV and Gamma-Ray Space Telescope Systems, (11 October 2004); doi: 10.1117/12.551915, 2004

[3] Hudec, R., Pína, L., Inneman, A., Švéda, L., LOBSTER - Astrophysics with Lobster Eye Telescopes, in Exploring the Cosmic Frontier, ESO Astrophysics Symposia European Southern Observatory 2007, pp.73-74, 2007

[4] Angel, J. R. P., 1979, Astroph. J., 364, 233

[5] Inneman, A., et al., 2000, Proc. SPIE, 4138, 94

[6] Hudec, R., et al., 2000, SPIE Proc. 4012, 432

[7] Hudec, R., et al., 2003, SPIE Proc. 4851, 578

[8] Hudec, R., et al., 2004a, SPIE Proc. 5488, 449

[9] Hudec, R., et al., 2004b, Nucl. Phys. B Proc. Suppl. 132, 320

[10] Ocalaghan, F. G., K. G. Henize, and J. D. Wray: SKYLAB ultraviolet stellar astronomy experiment S019, Applied Optics, Volume 16, id.973 (1977)

[11] Morgan, T. H., G. G. Spear, Y. Kondo, and K. G. Henize: Ultraviolet spectrophotometry from Gemini 11 of stars in Orion, The Astrophysical Journal, Volume 197, id.371 (1975)

[12] Urban, M., O. Nentvich, V. Stehlikova, T. Baca, V. Daniel, and R. Hudec: VZLUSAT-1: Nanosatellite with miniature lobster eye X-ray telescope and qualification of the radiation shielding composite for space application, Acta Astronautica, Volume 140, id.96 (2017) 
[13] Nentvich, O., M. Urban, V. Stehlikova, L. Sieger, and R. Hudec: Lobster eye X-ray optics: Data processing from two 1D modules, Contributions of the Astronomical Observatory Skalnate Pleso, Volume 47, id.178 (2017)

[14] Urban, M., O. Nentvich, V. Stehlikova, and L. Sieger: Detection of X-ray spectra and images by Timepix, Contributions of the Astronomical Observatory Skalnate Pleso, Volume 47, id.151 (2017)

[15] Nentvich, O., V. Stehlikova, M. Urban, R. Hudec, and L. Sieger: Data processing from lobster eye type optics, Society of Photo-Optical Instrumentation Engineers (SPIE) Conference Series, Volume 10235, id.102350N (2017)

[16] Daniel, V., L. Pina, A. Inneman, et al.: Terrestrial gamma-ray flashes monitor demonstrator on CubeSat, CubeSats and NanoSats for Remote Sensing, Volume 9978, id.99780D (2016)

[17] Schilling, K., Design of Pico-Satellites for Education in Systems Engineering. In: IEEE Aerospace and Electronic Systems Magazine 21 (2006), S. 9-14, 2006

[18] Schmidt, M., Ravandoor, K., Kurz, O., Busch, S., Schilling, K., Attitude Determination for the Pico-Satellite UWE-2. In: Space Technology 28. 2009, pp.67-74, 2009

[19] Schmidt, W. K. H., 1975, NucIM, 127, 285

[20] Švéda, L., et al., 2004, SPIE Proc. 5168, 393

[21] Tichý, V., et al., 2009, Balt. Astr. 18, 362

[22] Tichý, V., et al., 2011, Nucl. Instr. Meth. A, A633, S169

[23] Tichý, V., et al., 2013a, SPIE Proc. 8777, 877711

[24] Tichý, V., 2013b, SPIE Proc. 8777, 877710 